\title{
Lymphoid Stress Surveillance Response Contributes to Vitiligo Pathogenesis
}

\author{
Liisi Raam ${ }^{1,2}$, Epp Kaleviste ${ }^{3}$, Marina Šunina ${ }^{3}$, Helen Vaher ${ }^{3}$, Mario Saare $^{3}$, Ele Prans ${ }^{3}$, \\ Maire Pihlap ${ }^{3}$, Kristi Abram ${ }^{1,2}$, Maire Karelson ${ }^{1,2}$, Pärt Peterson ${ }^{3}$, Ana Rebane ${ }^{3}$, \\ Kai Kisand $^{3 *}$ and Külli Kingo ${ }^{1,2 *}$ \\ ${ }^{1}$ Department of Dermatology, University of Tartu, Tartu, Estonia, ${ }^{2}$ Dermatology Clinic, Tartu University Hospital, Tartu, \\ Estonia, ${ }^{3}$ Institute of Biomedicine and Translational Medicine, University of Tartu, Tartu, Estonia
}

\section{OPEN ACCESS}

Edited by:

Anne Davidson,

Feinstein Institute for Medical

Research, United States

Reviewed by:

Victoria Patricia Werth,

University of Pennsylvania,

United States

Jillian M. Richmond,

University of Massachusetts Medical

School, United States

*Correspondence:

Kai Kisand

kai.kisand@ut.ee

Külli Kingo

Kylli.Kingo@kliinikum.ee

Specialty section:

This article was submitted to

Autoimmune and Autoinflammatory

Disorders,

a section of the journal

Frontiers in Immunology

Received: 12 July 2018 Accepted: 01 November 2018 Published: 20 November 2018

Citation:

Raam L, Kaleviste E, Šunina M, Vaher $H$, Saare M, Prans E, Pihlap M, Abram K, Karelson M, Peterson P. Rebane $A$, Kisand $K$ and Kingo $K$ (2018) Lymphoid Stress Surveillance

Response Contributes to Vitiligo

Pathogenesis.

Front. Immunol. 9:2707.

doi: 10.3389/fimmu.2018.02707
Vitiligo is a chronic multifactorial depigmentation disorder characterized by the destruction and functional loss of melanocytes. Although a direct cytotoxic T cell attack is thought to be responsible for melanocyte damage, the events leading to the loss of self-tolerance toward melanocytic antigens are not understood. This research aimed to identify novel cellular and molecular factors that participate in vitiligo pathogenesis through the application of gene expression and immunofluorescence analysis of skin biopsy samples along with immunophenotyping of circulating cells. Our study provides insights into the mechanisms involved in melanocyte destruction. The upregulation of stress-ligand MICA/MICB, recognized by activating receptors on innate and innate-like T cells, imply involvement of lymphoid stress surveillance responses in vitiligo lesions. A simultaneous increase in the expression of transcription factor EOMES that is characteristic for innate-like virtual memory $T$ cells, suggest a similar scenario. Local lymphoid stress surveillance has been previously associated with the amplification of systemic humoral responses that were mirrored in our study by increased $T$ follicular helper cells and switched memory B cell proportions in patients with active vitiligo. In addition, microtubule-associated protein light chain 3 staining was compatible with the activation of autophagy in keratinocytes and in the remaining melanocytes of vitiligo lesional skin.

Keywords: vitiligo, interferons, MICA/MICB, WIPI1, LC3, EOMES, B cells, autophagy

\section{INTRODUCTION}

Vitiligo is an acquired multifactorial skin pigment disorder manifesting mainly as white macules on the skin due to melanocyte destruction. It is the most common depigmentation disorder affecting $0.5-1 \%$ of the population worldwide (1). Although depigmentation rarely causes any physical symptoms, vitiligo may cause psychosocial problems and impair quality of life (2). In addition, several comorbidities including autoimmune, systemic, and dermatologic diseases have been described (3).

Multiple mechanisms are supposed to be involved in melanocyte disappearance including genetic predisposition, environmental triggers, metabolic abnormalities, impaired renewal of melanocytes, and altered inflammatory and immune responses (4). Susceptibility to vitiligo is determined by several gene loci, most of which encode immunoregulatory factors and proteins 
involved in melanocyte function (5). As elevated levels of reactive oxygen species have been observed in vitiligo lesional skin and in melanocytes isolated from the skin of vitiligo patients (6), it is believed that in genetically susceptible individuals, environmental factors such as ultraviolet radiation, chemical agents, or mechanical trauma may lead to uncontrolled production of reactive oxygen species in the skin and consequently activate autoimmune processes resulting in melanocyte destruction (4). In addition to melanocyte dysfunction, alterations in keratinocytes also play a role. Keratinocytes in depigmented epidermis are more vulnerable to apoptosis and produce lesser amounts of melanogenic mediators than in normal skin. Furthermore, expression of genes involved in keratinocyte differentiation and cornification is dysregulated in lesional epidermis $(7,8)$.

Increased oxidative stress and alterations in both melanocytes and keratinocytes can induce production of pro-inflammatory cytokines and signals important for the activation of pathogenic immune cells in vitiligo patients $(4,9)$. It is thought that activation of the innate immune system through pathogen recognition receptors is impaired, which then induces autoimmunity in genetically predisposed patients (4). Melanocyte-specific antibodies, which are uncommon in healthy persons, have been found to circulate in the blood and deposit in the skin of vitiligo patients (10). CD8+ and CD4+ $\mathrm{T}$ cells are consistently found at the edge of actively depigmenting skin (11). Moreover, circulating skin-homing melanocyte-specific cytotoxic $\mathrm{T}$ lymphocytes have been found in the blood of vitiligo patients (12-15). However, the initial events that breach self-tolerance to melanocyte-specific antigens still remain unidentified.

This study aimed to identify the novel cellular and molecular patterns that determine vitiligo pathogenesis. Our results are consistent with the involvement of IFNs (16), and point to the activation of lymphoid stress surveillance responses and autophagy in diseased skin, but also strongly suggest the participation of germinal center reaction during the active phase of vitiligo.

\section{MATERIALS AND METHODS}

\section{Study Sample}

A case-control study was conducted in Dermatology Clinic, Tartu University Hospital. Ethical approval was obtained from the Research Ethics Committee of the University of Tartu. All the participants signed a written informed consent. We recruited 21 patients with non-segmental vitiligo (6 males, 15 females; age range 19-60 years) and 28 control subjects ( 8 males, 20 females; age range $24-57$ years) for the study. Vitiligo patients were recruited from the outpatient department of the Dermatology Clinic, Tartu University Hospital. The diagnosis of vitiligo based on the loss of pigmentation with typical localization and depigmented macules on the skin under Wood's lamp. Seven of the patients had active and 14 had stable vitiligo. Active vitiligo was defined as a condition in which development of new lesions or extension of old lesions was revealed in 3 months before examination. None of the patients had received any specific treatment for at least a month prior to the study. Control subjects were recruited from among health care personnel, medical students, and patients who turned to the dermatologic outpatient clinic for excision of naevi. They were free from chronic dermatoses and a positive family history of vitiligo. All of the participants were unrelated individuals of Caucasian race living in Estonia. For all the participants, form on the sociodemographic and clinical data was filled. Characteristics of the participants are shown in Table S1.

One skin punch biopsy sample (3-4 $\mathrm{mm}$ in diameter) from non-sun-exposed skin was taken from all control subjects. Two skin punch biopsy samples (3-4 $\mathrm{mm}$ in diameter) were collected from 16 vitiligo patients, one from the marginal zone of involved skin (hereinafter lesional skin) and another from non-sunexposed uninvolved skin (hereinafter non-lesional skin). The marginal zone of involved skin was chosen for gene expression study as inflammatory cell infiltration locates predominantly in the area between lesional and non-lesional vitiligo skin (10). For immunofluorescence assay, skin from the center of the vitiligo lesion and non-lesional skin was biopsied from 3 patients with vitiligo and embedded in OTC formulation. All the skin samples were instantly frozen in liquid nitrogen and stored at $-80^{\circ} \mathrm{C}$ until RNA extraction and immunofluorescence analysis.

From 20 vitiligo patients and 24 control subjects, $16 \mathrm{ml}$ venous blood was collected into $\mathrm{BD}$ Vacutainer ${ }^{\circledR} \mathrm{CPT}^{\mathrm{TM}}$ Cell Preparation Tubes with sodium heparin (BD Biosciences, Franklin Lakes, New Jersey, USA) and to separate plasma and peripheral blood mononuclear cells (PBMCs) from other blood cells the tubes were centrifuged at $1,500 \mathrm{~g}$ for $30 \mathrm{~min}$. Plasma was collected and stored at $-20^{\circ} \mathrm{C}$. Isolated PBMCs were washed twice with phosphate-buffered saline (PBS) and were stored in freezing medium in liquid nitrogen for flow cytometry.

\section{RNA Purification and qRT-PCR}

Gene expression analysis was carried out on skin biopsy samples of 16 vitiligo patients. 24 control subjects were included in this analysis. A total RNA was isolated from the skin using RNeasy Fibrous Tissue Mini Kit (Qiagen, Valencia, California, USA) or miRNeasy Mini Kit (Qiagen, Valencia, California, USA) according to the manufacturer's instructions. For RNA extraction the skin biopsy samples were homogenized in $700 \mu \mathrm{l}$ of the QIAzol Lysis Reagent (Qiagen, Valencia, California, USA) by a gentleMACS ${ }^{\mathrm{TM}}$ Dissociator (Miltenyi Biotec, Heidelberg, Germany) using $M$ tubes. The concentration and quality of the RNA were assessed with a NanoDrop ND1000 spectrophotometer (Thermo Fisher Scientific, Wilmington, Massachusetts, USA). cDNA was synthesized from 5000 ng of total RNA using oligo-dT and SuperScript ${ }^{\circledR}$ III Reverse Transcriptase (Life Technologies, Carlsbad, California, USA) according to the manufacturer's instruction.

For amplification of the PCR product, SYBR ${ }^{\circledR}$ Green (Life Technologies, Carlsbad, California, USA) master mix was used. qRT-PCR analysis was carried out on ViiA ${ }^{\mathrm{TM}} 7$ Real-Time PCR system (Life Technologies, Carlsbad, California, USA). Primer sequences are listed in Table S2. The relative gene expression levels were calculated using the comparative $\mathrm{Ct}(\Delta \Delta \mathrm{Ct})$ method and normalized to the expression of Actin, Beta (ACTB). 


\section{Cytokine Testing}

The concentration of proteins TNF- $\alpha$, IL-1b, IL-1Ra, IL-2, IL-5, IL-6, CXCL8, CXCL10, IFN- $\gamma$, Granulocyte-Colony Stimulating Factor (G-CSF) and Granulocyte-Macrophage-(GM)-CSF was measured in plasma of 18 patients with vitiligo and 24 control subjects. The xMAP technology on Luminex 200 (Luminex Corporation, Austin, Texas, USA) was used. The Milliplex MAP multiplex assay was conducted in a 96-well microplate format according to the manufacturer's instructions (Millipore, Billerica, Massachusetts, USA).

\section{Flow Cytometry}

Surface marker expression on PBMCs from 17 vitiligo patients and 18 control subjects was assessed by flow cytometry. Cells were stained in flow cytometry staining buffer (PBS with $0.5 \%$ bovine serum) for $20 \mathrm{~min}$ at $4^{\circ} \mathrm{C}$ with antibodies listed in Table S3. Stained cells were analyzed using LSRFortessa flow cytometer (BD Biosciences) and FACSDiva version 6 (BD Biosciences) software. The optical detector configuration is provided in Table S4.

\section{Cell Isolation and Culture}

Pooled, normal human epidermal keratinocytes (Promocell, Heidelberg, Germany) were cultured in Keratinocyte-SFM medium with supplements (Life Technologies, Carlsbad, California, USA) at $37^{\circ} \mathrm{C}$ in $5 \%$ humidified $\mathrm{CO}_{2}$ incubator. For two-dimensional (2D) cultures, cells with density of 20,000 cells per well on 24-well plates were seeded and for 3D keratinocyte culture in air liquid interface, 50,000 cells per well were seeded on ThinCert Cell Culture Inserts $(0.4 \mu \mathrm{m}$ pore, $0.33 \mathrm{~cm} 2$ ) on 24-well plates (Greiner Bio-One, Kremsmünster, Austria). For the 3D culture, Keratinocyte-SFM medium with supplements and Dulbecco's Modified Eagle Medium (both from Life Technologies, Carlsbad, California, USA) containing High Glucose, GlutaMAX ${ }^{\mathrm{TM}}$ and Pyruvate in 1:1 ratio were used. For the 2D culture, Keratinocyte-SFM medium with supplements (Life Technologies, Carlsbad, Califonia, USA) was used. Melanocytes and fibroblasts were isolated and cultured as described by Reemann et al. (17).

Monocyte derived Langerhans cells (moLC) were generated as follows. PBMCs were isolated from buffy coat by Ficoll-Hypaque Plus (Amersham Biosciences, Piscataway, USA) density gradient centrifugation. Monocytes were isolated using MACS anti-CD14 beads (Miltenyi Biotec, Bergisch Gladbach, Germany) according to the manufacturer's protocol up to purity over $95 \%$. The isolated cells were cultured in RPMI 1640 medium (supplemented with $10 \%$ fetal calf serum, $1 \%$ penicillin and streptomycin, all from PAA Laboratories, Pasching, Austria) at a density of 1-1.5 × 106 cells $/ \mathrm{ml}$. moLC were differentiated for seven days in the presence of GM-CSF (50 ng/ml), IL-4 (25 ng/ml), TGF $(10 \mathrm{ng} / \mathrm{ml})$, and TSLP ( $5 \mathrm{ng} / \mathrm{ml}$ ), all from R\&D Systems, Minneapolis, USA (18).

\section{Immunofluorescence}

Immunofluorescence was performed on $5 \mu \mathrm{m}$ thick frozen sections of skin biopsy samples. The samples were fixed with $4 \%$ formaldehyde and permeabilized with $0.2 \%$ Triton X-100 in PBS. After that, the slides were stained using the Alexa Fluor ${ }^{\mathrm{TM}} 594$
Tyramide SuperBoost ${ }^{\text {TM }}$ Kit and goat anti-mouse IgG (Thermo Fisher Scientific, Wilmington, Massachusetts, USA) according to the manufactures manual. Briefly, the slides were blocked $60 \mathrm{~min}$ at room temperature (RT) with $10 \%$ normal goat serum and then incubated with primary antibodies for $60 \mathrm{~min}$ at RT. The used antibodies were mouse monoclonal antibody to microtubuleassociated protein light chain 3 (LC3) (nanoTools, Teningen, Germany) and mouse anti-human MICA/MICB antibody (BioLegend, San Diego, California, USA). After that the slides were incubated $60 \mathrm{~min}$ at RT with poly-HRP-conjugated goat anti-mouse secondary antibody. For the signal enhancement, the tyramide working solution was added for $6 \mathrm{~min}$ at RT and the stop solution was used to halt the HRP reaction. To identify melanocytes, the sections were incubated with antiTYRP1 (tyrosinase related protein 1) rabbit polyclonal antibody (Atlas Antibodies, Sweeden) at $4^{\circ} \mathrm{C}$ overnight and incubated with Alexa Fluor ${ }^{\circledR} 488$ conjugated Goat anti-Rabbit IgG $(\mathrm{H}+\mathrm{L})$ Secondary Antibody (1:1,000, ThermoFisher Scientific, 1:500) for $60 \mathrm{~min}$ at RT. After nuclear counterstain with DAPI (4',6-Diamidine-2' -phenylindole dihydrochloride, $1 \mu \mathrm{g} / \mathrm{mL})$ for $10 \mathrm{~min}$ the slides were washed three more times in PBS and covered with fluorescent mounting medium (Dako, Santa Clara, California, USA) and coverslips. Images were obtained with FV1200 confocal microscope (Olympus, Tokyo, Japan). For Figure 5E, the fluorescence signal marking LC3 and DAPI were quantified using Fiji (19) with built-in options.

\section{Statistical Analysis}

Statistical analysis was performed using the R statistical software (https://www.r-project.org/). The mRNA expression values and the fluorescence signal ratios were log-transformed before the statistical testing to adhere with the assumptions of the normal distribution. The conformity to a normal distribution was assessed using the Kolmogorov-Smirnov test. Comparisons between vitiligo patients and control subjects were made using the unpaired Student's $t$-test. Comparing vitiligo lesional skin with non-lesional skin, the paired Student's $t$-test was used. In mRNA expression analysis and immunofluorescence signal quantification, if vitiligo lesional and non-lesional skin was compared to healthy control skin, the Dunnett's correction was applied to adjust for multiple comparisons. The mean and standard deviation of the log-transformed mRNA expression values were back-transformed to linear scale for plotting, which is shown as the geometric mean $\times \div$ geometric standard deviation on the graphs. Correlation was assessed with the Pearson's correlation coefficient. A $p$-value $<0.05$ was considered significant.

\section{RESULTS}

\section{Lesional Vitiligo Skin Is Characterized by an Interferon Signature}

Although vitiligo lesional skin does not manifest substantial macroscopic signs of inflammation, the processes that occur at the cellular and molecular level should leave some informative traces to local gene expression that would help dissect the processes leading to melanocyte destruction. Like multiple 
previous studies $(7,20-23)$ we could only detect very moderate upregulation of some inflammatory cytokines such as TNF $(p<0.05)$ and IL36A (alias IL1F6) $(p<0.05)$ in vitiligo lesional or non-lesional skin (Figure 1A). The transcripts of IFNG and Th17 specific cytokines remained undetectable in control as well as in vitiligo biopsy samples. In addition, IL-1 inhibitory IL1RN $(p<0.05)$ showed a slight increase in vitiligo non-lesional skin. From the studied chemokines, only CCL5 $(p<0.05)$ and CXCL10 $(p<0.001)$ were significantly upregulated in vitiligo lesional skin compared with healthy control skin (Figure 1A). CXCL10 is an interferon (IFN) regulated gene like IFIH1 (24), which was also upregulated in vitiligo lesional $(p<0.01)$ and in non-lesional skin $(p<0.05)$ (Figures 1A,B). From the studied cytokine receptors, only IL22RA1 $(p<0.05)$ expression was increased in the non-lesional skin of vitiligo patients compared with the skin of control subjects (Figure 1B). Whether this implicates the IL-22 pathway in vitiligo pathogenesis remains unknown as IL22 transcripts were below the detection limit. However, upregulation of IL22 in the PBMCs of vitiligo patients has been reported earlier (25). From the studied inflammasome-related genes only AIM2 was slightly elevated in vitiligo non-lesional skin (Figure 1C). As the vitiligo group consisted of patients with active as well as stable disease, it remained possible that the differences between groups could be masked by the high proportion of patients without an active process in their lesions. Therefore, we compared the gene expression between patients with active or stable disease; however, the data did not reveal higher inflammatory cytokine expression in active vitiligo skin (Table S5).

To check for the possible signs of systemic inflammation, we measured the concentration of inflammation-associated cytokines (TNF- $\alpha$, IFN- $\gamma$, IL-1b, IL-1Ra, IL-2, IL-5, IL-6, CXCL8, CXCL10, G-CSF, and GM-CSF) in the plasma of vitiligo patients. We found slightly lower plasma concentrations of IL-1 inhibitory IL1-Ra $(p<0.05)$ in vitiligo patients compared to those in control subjects, and also less G-CSF $(p<0.01)$ (Figure 2). G-CSF may have pro-inflammatory or pro-repair influence depending on the context, and therefore the impact of this change is difficult to judge (26). To conclude, these results further support that strong systemic inflammation is probably not a part of vitiligo pathogenesis.
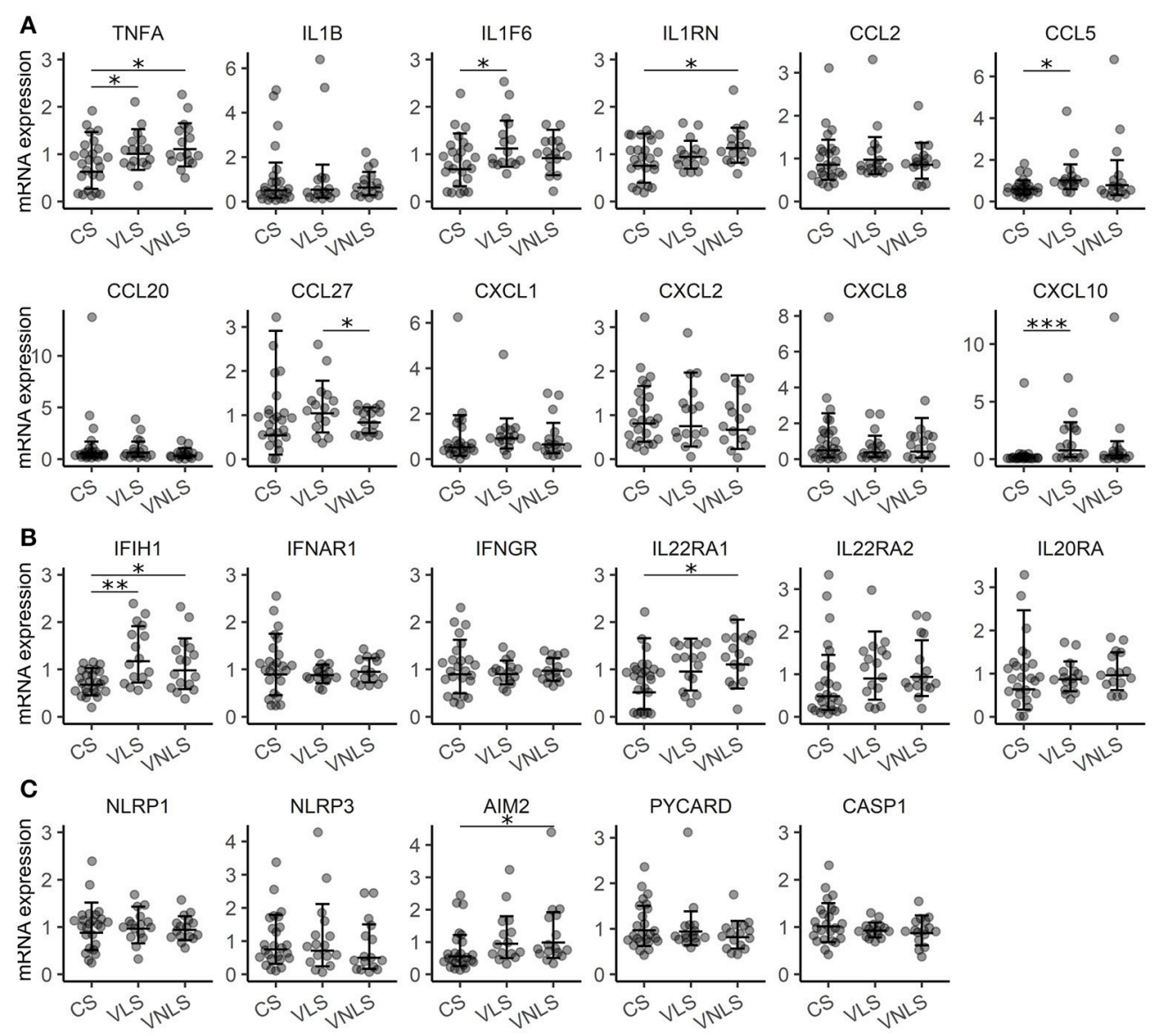

FIGURE 1 | Gene expression signature is consistent with a very mild inflammatory response in vitiligo. Relative expression of mRNAs encoding (A) cytokines, (B) receptors and $\mathbf{( C )}$ components of the inflammasome in the skin of control subjects (CS), and in the lesional skin (VLS) and non-lesional skin (VNLS) of vitiligo patients. Geometric mean $\times \div$ geometric standard deviation is indicated. ${ }^{*} p<0.05$; ${ }^{* *} p<0.01$; and ${ }^{* * *} p<0.001$. 


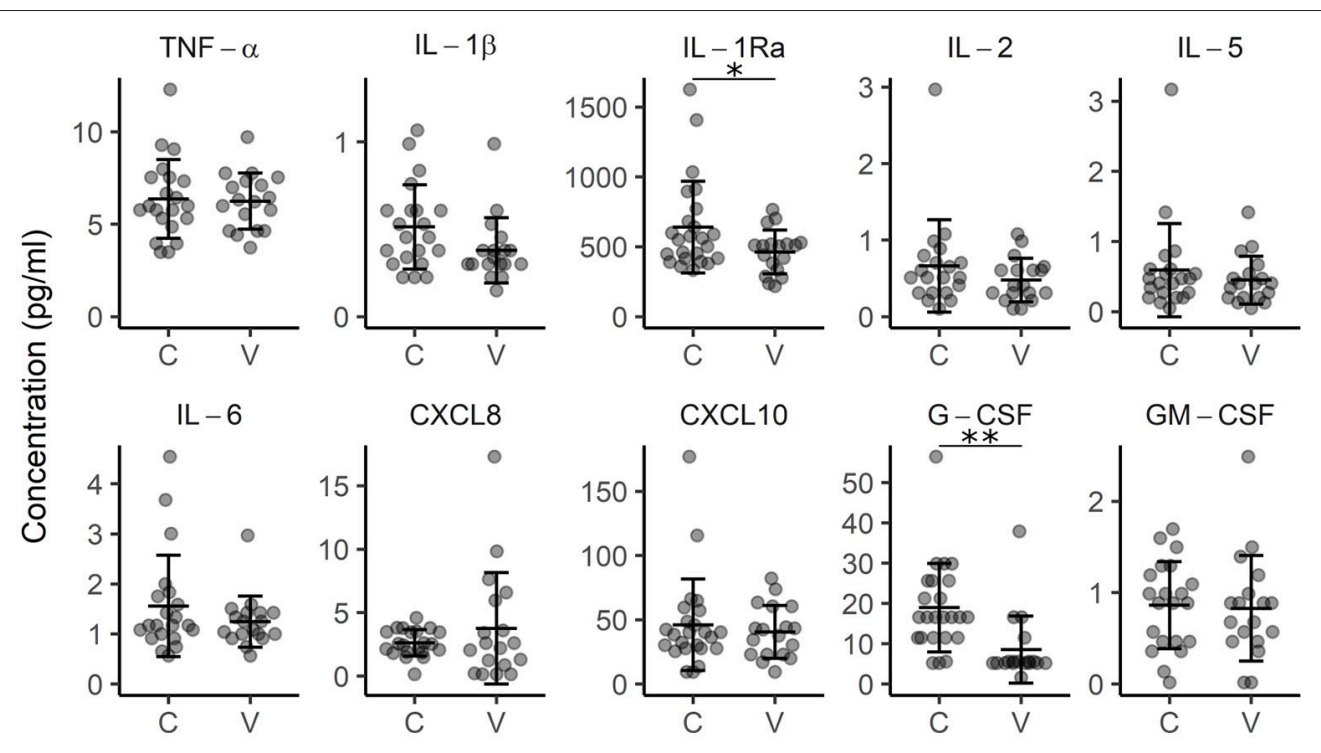

FIGURE 2 | Concentration of cytokines in the plasma of control subjects $(C)$ and vitiligo patients $(\mathrm{V})$. Arithmetic mean \pm standard deviation is indicated. ${ }^{*} p<0.05$; ${ }^{* *} p<0.01$.

\section{Innate-like Cells may be Responsible for Vitiligo Lesions}

Next, we analyzed immune cell infiltration to vitiligo lesions based on cell-type specific gene expression. In particular, we were interested in cells with cytotoxic potential toward melanocytes, and also in cells capable of counterbalancing immune responses. We did not find increased FOXP3 levels in vitiligo skin, indicating comparable numbers of Tregs in the studied groups (Figure 3A). However, the expression of CTLA4 was increased in vitiligo lesional and non-lesional skin $(p<0.001$ and $p<0.001$, respectively) (Figure 3A). As CTLA4 is expressed in Tregs as well as in activated $\mathrm{T}$ cells, we suggest that CTLA4 is upregulated in our samples due to increased activation of $\mathrm{T}$ cells in the skin of vitiligo patients. Moreover, EOMES, a transcription factor characteristic for effector cytotoxic $\mathrm{T}$ cells and innate and innate-like virtual memory $\mathrm{T}$ cells, was increased in both vitiligo lesional and non-lesional skin $(p<0.001$ and $p<0.001$, respectively) (Figure 3A) $(27,28)$. KLRK1, encoding the activating NK cell receptor NKG2D and TRGC1 that encodes a $\mathrm{T}$ cell receptor $\gamma$-chain showed a tendency for increased expression in vitiligo lesional skin compared to that in healthy skin ( $p=0.055$ and $p=0.058$, respectively) (Figure 3A). Notably, the stress molecule $\mathrm{MHC}$ class 1 chain-related protein $\mathrm{A}$ and $\mathrm{B}$ (MICA/MICB) that can be bound by activating $\mathrm{NK}$ cell receptors also showed a tendency for increased expression in vitiligo lesions $(p=0.052$ ) (Figure 3A). Next we performed MICA/MICB immunofluorescence staining on sections from vitiligo and control biopsy samples together with the melanocyte marker TYRP1 (Figure 3B, Figure S1). While control skin and vitiligo non-lesional skin were completely clean of MICA/MICB, we noted multiple positive cells in the sub-epidermal area of vitiligo lesional skin. Often the stained cells were with disturbed nuclear morphology, as can be seen in the middle panel of Figure 3B. There was no co-localisation of MICA/MICB with
TYRP1. The much more dramatic differences in MICA/MICB protein expression in comparison to gene expression data can be explained by the posttranscriptional and posttranslational regulation of stress molecules (29). To conclude, the data suggest the importance of stress-ligand upregulation in the dermis of vitiligo lesional skin and the presence of activated innate-like $\mathrm{T}$ cells at the center of the pathological process indicating the involvement of lymphoid stress surveillance responses in vitiligo pathogenesis.

\section{Active Vitiligo Is Characterized by Increased Proportion of Circulating Memory B Cells}

Next, we applied flow cytometry to find out whether any signs of immune cell dysregulation could be seen among the circulating lymphocyte subpopulations. The gating strategy for various subpopulations of classical and non-classical T cells as well as B cells is depicted in Figure S2. Using CCR7, CD45RA and CD28, $\mathrm{CD} 4+$ and $\mathrm{CD} 8+\mathrm{T}$ cells were divided into subpopulations at different levels of maturation. These subpopulations as well as the percentages of $\mathrm{V} \delta 1 \mathrm{TCR}+$ and $\mathrm{V} \delta 2 \mathrm{TCR}+\gamma \delta \mathrm{T}$ cells, mucosa associated invariant $\mathrm{T}$ cells, and $\mathrm{NK}$ cells did not demonstrate significant differences between the studied groups (Table S6). We could identify only a moderate decrease in the percentage of Tregs ( $p<0.05$ ) confirming previous findings (30) but also an increased proportion of unswitched memory (USM) B cells (Figure 4A). When vitiligo patients were divided into subgroups according to their disease activity, it was evident that active disease was associated with decreased percentages of naïve B cells and increased proportions of switched memory (SM) and USM B cells (Figure 4B, Table S7). The reduction of naïve B cells does not probably stem from impaired generation of $B$ cells in the bone marrow as the percentages of transitional $\mathrm{B}$ cells mostly remained 


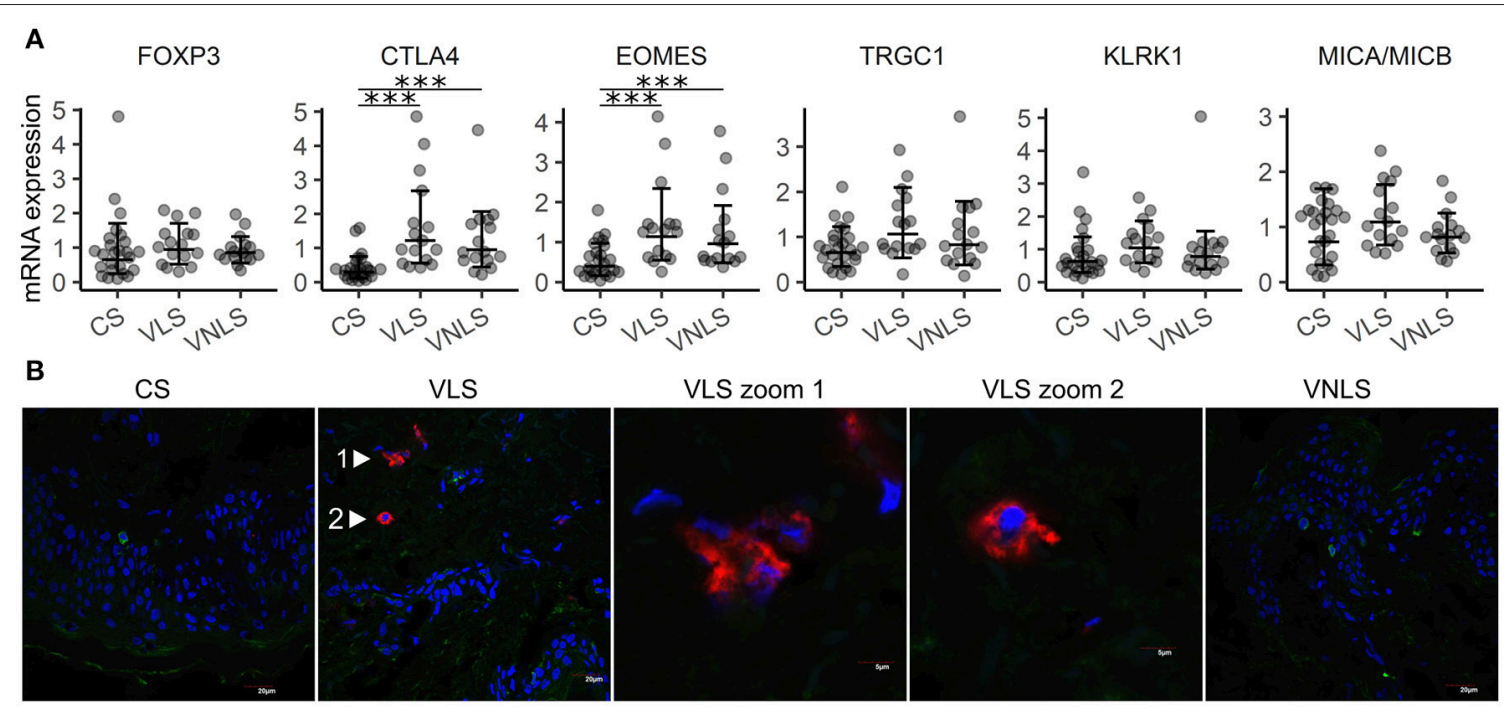

Stainings: MICA/MICB, TYRP1 and DAPI

FIGURE 3 | Innate-like cells and receptors are involved in vitiligo pathogenesis. (A) Relative mRNA expression of genes encoding markers of infiltrating immune cells and their target molecules. Geometric mean $x \div$ geometric standard deviation is indicated. ${ }^{* *} p<0.001$. (B) Immunofluorescence image of MICA/MICB (MHC class I chain-related protein A and B) and TYRP1 (tyrosinase related protein 1) in sections of biopsy samples from control subjects (CS), and in the lesional skin (VLS) and non-lesional skin (VNLS) of vitiligo patients. The images are representative of 3 healthy controls and 3 vitiligo patients.

unchanged. Instead, activation of germinal centers and faster B cell isotype switching is supported by concomitant upregulation of $\mathrm{T}$ follicular helper (Tfh) cell percentages in patients with active vitiligo (Figures 4B,C). To conclude, our results point to the involvement of $\mathrm{B}$ cells and germinal center reactions in vitiligo pathogenesis, although the disease is believed to be mediated by cytotoxic T cells.

\section{WIPI1 Is Significantly Downregulated in Vitiligo Skin}

According to several hypotheses of vitiligo pathogenesis, melanocytes are not merely innocent bystanders that are attacked by cytotoxic cells, but rather trigger the processes themselves by impaired oxidative stress responses and/or impairment of autophagic processes. Therefore, we set out to study the expression of WIPI1 that is a gene regulating autophagy and melanosome maturation $(31,32)$. Its expression was significantly downregulated in both lesional $(p<0.01)$ and non-lesional $(p<0.001)$ skin taken from patients with vitiligo (Figure 5A). We compared WIPI1 mRNA expression levels in various cell types present in the skin and found the highest expression level in melanocytes that was followed by fibroblasts (Figure 5B). WIPI1 expression was relatively low in monocyte derived Langerhans cells and keratinocytes in 2-dimensional culture but was increased during their differentiation at the air-liquid interface. Regarding the highest contribution of WIPI1 expression from melanocytes in the biopsy samples, WIPI1 downregulation could be the result of melanocyte loss in the lesions. However, this is unlikely regarding the reduced WIPI1 expression in vitiligo non-lesional skin.

To determine if WIPI1 downregulation in vitiligo skin can be associated with impaired autophagy in melanocytes, we stained the tissue sections of skin biopsy samples for microtubule-associated protein light chain 3 (LC3) (Figure 5C). When autophagy is initiated, the cytosolic form of LC3 is conjugated to phosphatidylethanolamine to form a lipidated LC3 conjugate, which is recruited to autophagosomal membranes. This is associated with the conversion of the dim homogenous fluorescence to a dotted pattern in the cytosol. In control skin and in vitiligo non-lesional skin, most of the melanocytes were devoid of LC3 dots, whereas in the vitiligo lesions, the scarce remaining melanocytes with weak TYRP1 staining showed an LC3 pattern characteristic for autophagy (Figure 5C, Figure S3). To our surprise, keratinocytes in vitiligo lesions also had significantly more autophagic vacuoles throughout all the layers of epidermis, in comparison to control and non-lesional skin (Figures 5D-E, Figure S4). Autophagy and nucleophagy is thought to be essential for normal epidermal development and differentiation (32). To the best of our knowledge, this is the first description of excessive autophagy in vitiligo keratinocytes. However, previous transcriptional studies have found alterations in keratinocytes from vitiligo lesions (7). This suggests that the cross-talk between different skin cell types is relevant in vitiligo pathogenesis. Collectively, our results are in line with increased autophagy in vitiligo lesions, involving melanocytes as well as keratinocytes.

\section{DISCUSSION}

This study provided many insights into vitiligo pathogenesis. The results suggest the participation of lymphoid immune surveillance in vitiligo lesions, activation of germinal center reactions in secondary lymphoid organs during lesion extension, and support the involvement of autophagy in disease development. 

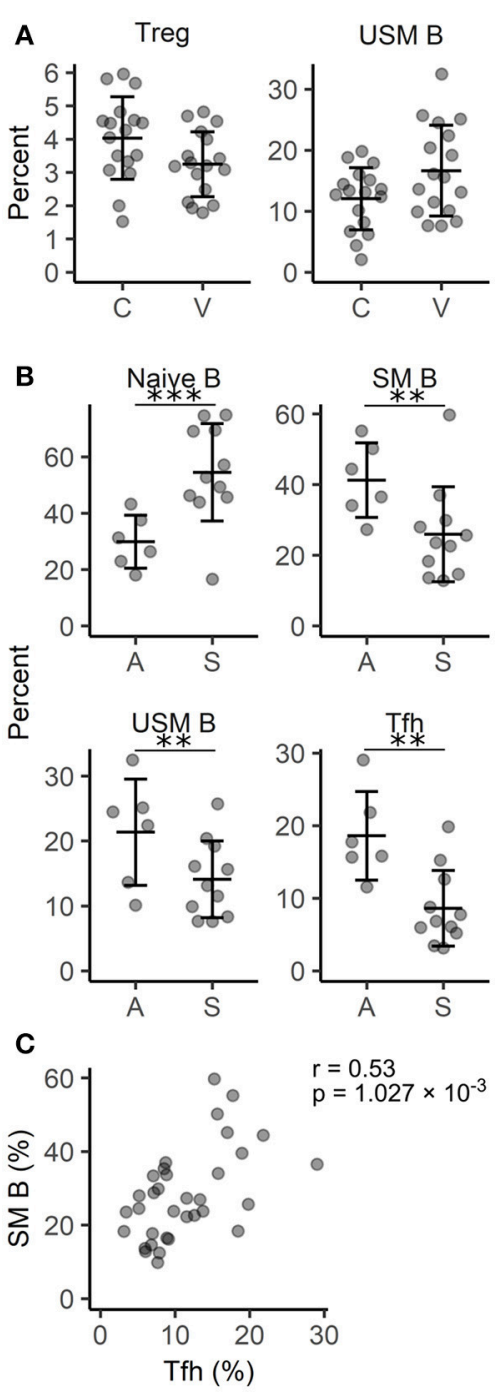

FIGURE 4 | Comparison of circulating lymphocyte subpopulations in vitiligo patients with different disease activity. (A) The percentage of regulatory $T$ cells (Treg) among T helpers and unswitched memory (USM) B cells among B cells in vitiligo patients $(\mathrm{V})$ and control individuals $(\mathrm{C})$. (B) The percentage of naïve, switched memory (SM) and USM B cells among B cells and follicular helper T cells (Tfh) among T helper cells in vitiligo patients with active (A) Vs. stable (S) disease. Arithmetic mean \pm standard deviation is indicated. ${ }^{*} p<0.05$; ${ }^{* *} p<0.01$; and ${ }^{* * *} p<0.001$. (C) A scatter plot showing the correlation between the percentages of SM B cells and Tfh cells. Pearson's correlation coefficient ( $r$ ) and the $p$-value of the association test are indicated on the plot.

Vitiligo lesions develop without severe clinical signs of inflammation. In line with this, the comparative gene expression study of skin biopsy samples revealed only a mild inflammatory response in the marginal areas of vitiligo lesions where the destructive processes occur. Among the studied inflammatory chemokines, only CXCL10 stood out as a factor that is significantly upregulated right in the center of the pathological processes. The importance of CXCL10 together with closely related CXCL9 (33) has been substantiated by previous studies in humans as well as in mouse models of vitiligo $(9,23,24,34)$.
Although CXCL10 is known as an IFN- $\gamma$-induced and Th1associated chemokine, CXCL10 expression is also influenced by type I IFNs (35). Indeed, the tissue-resident memory T (Trm) cells in vitiligo skin express CXCR3, the receptor for CXCL10, and are prone to IFN- $\gamma$ production $(12,14)$. However, the mRNA of type II IFN, IFN- $\gamma$, remained below the detection limit in our biopsy samples. In contrast, recent evidences suggest that type I IFNs play a role in vitiligo pathogenesis: IFN- $\alpha$ producing plasmacytoid dendritic cells infiltrate active vitiligo skin $(16,36)$; pegylated IFN- $\alpha 2 \mathrm{a}$ and IFN- $\alpha 2 \mathrm{~b}$, which are used in the treatment of chronic hepatitis $\mathrm{C}$, induce depigmentation at the injection sites (37), and vitiligo patches have been observed at the site of application of imiquimod, a Toll-like receptor (TLR)-7 and TLR8 agonist that enhances IFN- $\alpha$ production (16). In addition to CXCL10, IFN-induced IFIH1 was upregulated in vitiligo skin in the current study. Moreover, it has been previously observed that the intracellular innate immune receptor IFIH1 induces type I IFNs and that IFIH1 is implicated in autoimmune processes (38), and is one of the susceptibility loci/risk genes for vitiligo (5). During the course of vitiligo, type I IFNs can be induced by the cGAS-cGAMP-STING pathway after oxidative stress generated DNA damage $(39,40)$. To conclude, the results of the current study indirectly support the association between type I IFNs and vitiligo. Nevertheless, the specific and/or overlapping roles of type I and II IFNs in vitiligo have yet to be substantiated.

Our study also helps establish the link between lymphoid stress surveillance response and vitiligo. In addition to microbes, tissue dysregulation can be sensed by lymphocytes via recognition of stress molecules (like $\mathrm{MICA} / \mathrm{MICB}$ ) that have been upregulated by damaging agents like oxidative stress (29, 41). In lymphoid stress surveillance, lymphocytes are rapidly activated through their nonclonotypic receptors and deploy their effector mechanisms like cytokine secretion and cytotoxic mediators without any delays $(41,42)$. We could clearly observe staining for MICA/MICB in dermal areas of vitiligo lesional skin but not in healthy or non-lesional skin. Although melanocytes are situated at the bottom layer of the epidermis, dermal changes in vitiligo have been described by previous studies as well (43). MICA/MICB are ligands for the activating NKG2D receptor, expressed by NK cells, $\gamma \delta \mathrm{T}$ cells, and a subpopulation of cytotoxic $\alpha \beta$ T cells (42). Involvement of innate cells in vitiligo has been suggested by $\mathrm{Yu}$ et al. (44). We could not detect significant upregulation of TRGC1 that encodes a $\mathrm{T}$ cell receptor $\gamma$-chain arguing against the specific recruitment of $\gamma \delta \mathrm{T}$ cells to vitiligo lesions. However, this is a tissue resident population of innate-like $\mathrm{T}$ cells that is already located and ready to respond to stress signals by neighboring cells. Accordingly, the lack of increase in their numbers does not mean that they are not responding to the stress-ligands. However, the increase in transcription factor EOMES is consistent with the involvement of innate-like virtual memory $\mathrm{T}$ cells that like innate cells, respond readily to cytokines and stress signals, and importantly, can be recruited by CXCL10 $(27,28)$. Interestingly, it has been reported that NKG2D-dependent communication between dysregulated epithelial cells and tissue-associated lymphoid cells also affects the systemic immune compartment in mice (42). In that study, epithelial stress-surveillance enhanced the response 
A

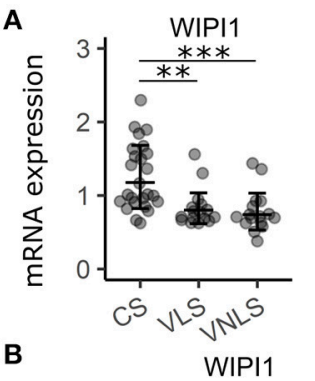

B

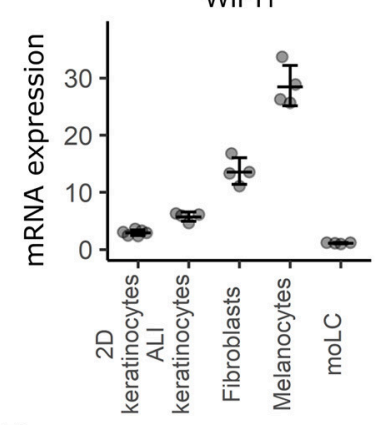

D

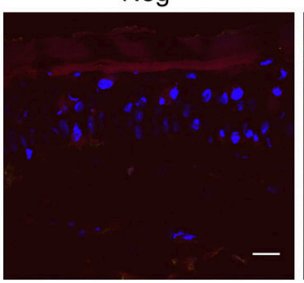

Stainings: LC3, TYRP1 and DAPI
C

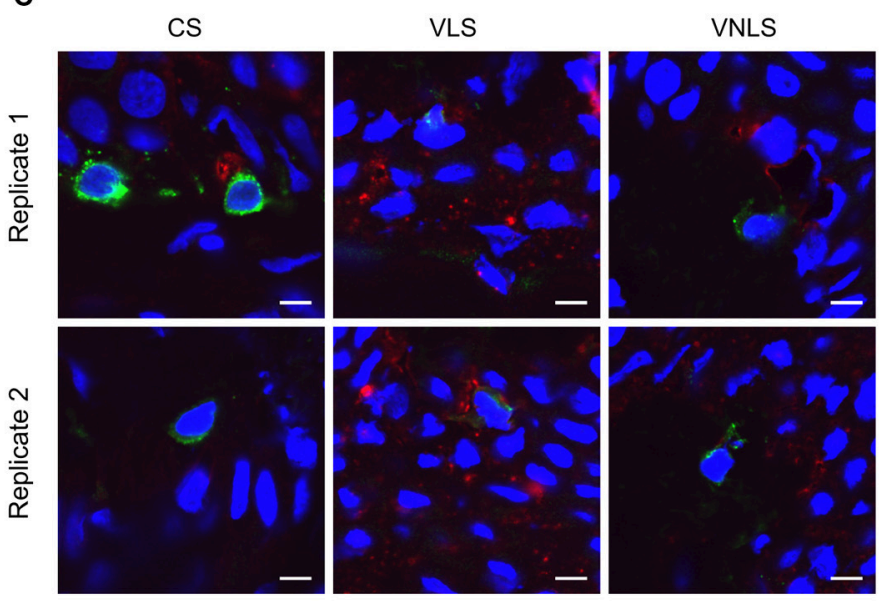

Stainings: LC3, TYRP1 and DAPI

FIGURE 5 | WIPI1 expression and LC3 staining. (A) WIPI1 relative mRNA expression in the skin of control subjects (CS), and in the perilesional skin (VLS) and non-lesional skin (VNLS) of vitiligo patients. Geometric mean $\times \div$ geometric standard deviation is indicated. ${ }^{* *} p<0.01$; and ${ }^{* * *} p<0.001$. (B) WIPI1 relative mRNA expression in various cell types of the skin. Geometric mean $\times \div$ geometric standard deviation is indicated. 2D, two-dimensional; ALI, air-liquid interface; moLC, monocyte derived Langerhans cells. (C) Immunofluorescence image of LC3 (microtubule-associated protein 1A/1B-light chain 3) and TYRP1(tyrosinase related protein 1) in sections of biopsy samples from control subjects (CS), and lesional skin (VLS) and non-lesional skin (VNLS) of vitiligo patients. The white bar represents $5 \mu \mathrm{m}$. (D) Immunofluorescence image as in panel $\mathrm{C}$ with the white bar representing $20 \mu \mathrm{m}$. Neg. refers to a control slide with secondary antibodies only. (E) The box and whiskers plots show the median and interquartile range of the ratio between the fluorescence signal marking LC3 expression and DAPI (cell nuclei) in CS, VNLS, and VLS. Whiskers cover data points within a $1.5 \times$ interquartile range. ${ }^{*} p<0.05$.

to antigens that were introduced together with epithelial stress, and provoked the $\mathrm{T}$ helper 2 and antibody responses linking lymphoid stress surveillance to atopy. These data from mouse models are echoed by clinical findings that atopic dermatitis is included among the vitiligo comorbidities (45). Moreover, in line with this information, we found significantly more switched and unswitched memory B cells and T follicular helpers in patients with active vitiligo compared to those in patients with stable disease (with good correlation between the percentages of SM $\mathrm{B}$ and Tfh). This suggests that although the damage is probably caused by local resident lymphocytes, the local processes may have more profound systemic effects than suspected so far. The involvement of humoral responses in vitiligo is corroborated by the finding of circulating autoantibodies directed toward melanocytic antigens whose levels correlate with disease activity as biomarkers of melanocyte destruction $(46,47)$. However, the precise cell type responsible for lymphoid stress surveillance in vitiligo and the molecular mechanisms that link the local processes in skin with systemic effects are yet to be identified.

Interestingly, we demonstrated the highly significant downregulation of a regulator of autophagy, WIPI1 (32), in lesional and non-lesional skin of vitiligo patients. Autophagy is the enzymatic digestion of cytoplasmic contents. In this process, cytoplasmic proteins are trapped within vesicles called autophagosomes, which fuse with lysosomes and the cytoplasmic proteins are proteolytically degraded. Although autophagy is primarily a mechanism for degrading damaged cellular organelles and proteins to maintain cellular homeostasis, it is shown to be involved in pathological processes including autoimmunity, infections, and malignant tumors (48, 49). Autophagy is controlled by macromolecular signaling complexes. Of these, Beclin 1 with UV radiation resistance-associated tumor suppressor gene protein (UVRAG) are positive regulators, and the mammalian target of rapamycin (mTOR) is a negative 
regulator of autophagy (48). The idea that dysfunction of autophagy is involved in vitiligo pathogenesis is supported by Jeong et al., who found that variation in UVRAG gene contributes to the risk of non-segmental vitiligo in the Korean population (50), and by Wang et al., who demonstrated that several genes involved in the autophagy process were dysregulated in leukocytes of generalized vitiligo patients (51). Moreover, autophagy deficiency causes premature senescence and decreased proliferation of melanocytes (52), and melanosomal autophagy in stressed melanocytes mediates antigen presentation and dendritic cell maturation (53). However, melanosomes are also lysosome-related organelles whose maturation is controlled by molecules overlapping with autophagy regulators like WIPI1 and LC3 $(31,54)$. Therefore, in vitiligo skin, WIPI1 downregulation may be rather linked with impaired maturation of melanosomes and not with impaired autophagy. According to our data, downregulation of WIPI1 in vitiligo skin and activation of autophagy are uncoupled processes as WIPI1 expression was reduced to a similar degree in lesional and non-lesional skin whereas LC3 staining clearly indicated increased autophagy only in keratinocytes of lesional skin. Whether the increased autophagy in vitiligo keratinocytes represents a compensatory mechanism for the lack of melanin, is responsible for the faster degradation of remaining melanin produced by residual melanocytes in vitiligo skin (55) or is the result of increased stress-surveillance response in the skin is still an open question.

Thus, we propose a model wherein different stressors like UV irradiation or dysregulated melanosome maturation lead to increased oxidative stress that can cause DNA damage. Damaged cells upregulate stress-ligands and type I IFN production. IFNs induce CXCL10 secretion from keratinocytes, which then attracts CXCR3 positive T cells. Stress-ligands can be bound by activating receptors on innate and innate-like $\mathrm{T}$ cells which unleash their effector mechanisms and cause autoantigen release from dying cells. This can prime antigen-specific $\mathrm{T}$ and $\mathrm{B}$ cell responses, which participate in the perpetuation of tissue damage.

\section{REFERENCES}

1. Ezzedine K, Eleftheriadou V, Whitton $M$ and van Geel N. Vitiligo. Lancet (2015) 386:74-84. doi: 10.1016/s0140-6736(14)60763-7

2. Silverberg JI, Silverberg NB. Association between vitiligo extent and distribution and quality-of-life impairment. JAMA Dermatol. (2013) 149:15964. doi: 10.1001/jamadermatol.2013.927

3. Dahir AM, Thomsen SF. Comorbidities in vitiligo: comprehensive review. Int J Dermatol (2018) 57:1157-64. doi: 10.1111/ijd.14055

4. Boniface K, Seneschal J, Picardo M, Taieb A. Vitiligo: focus on clinical aspects, immunopathogenesis, and therapy. Clin Rev Allergy Immunol. (2018) 54:52-67. doi: 10.1007/s12016-017-8622-7

5. Jin Y, Andersen G, Yorgov D, Ferrara TM, Ben S, Brownson KM, et al. Genome-wide association studies of autoimmune vitiligo identify 23 new risk loci and highlight key pathways and regulatory variants. Nat Genet. (2016) 48:1418-24. doi: 10.1038/ng.3680

6. Li SL, Zhu GN, Yang YQ, Jian Z, Guo S, Dai W, et al. Oxidative stress drives CD8(+) T-cell skin trafficking in patients with vitiligo through CXCL16 upregulation by activating the unfolded protein response in keratinocytes. $J$ Allergy Clin Immunol. (2017) 140:177-89. doi: 10.1016/j.jaci.2016.10.013

\section{ETHICS STATEMENT}

This study was carried out in accordance with the recommendations of Research Ethics Committee of the University of Tartu. All subjects gave written informed consent in accordance with the Declaration of Helsinki. The protocol was approved by the Research Ethics Committee of the University of Tartu.

\section{AUTHOR CONTRIBUTIONS}

LR, EP, and MP performed and analyzed the gene expression experiments, LR and MS performed the bioinformatic analysis of the data and prepared the figures, EK and MŠ designed and performed the flow cytometric experiments, HV performed immunofluorescence staining, LR and MP carried out Luminex experiments. LR, KüK, MK and KA sampled the patients and collected the clinical data. KaK, KüK, PP and AR supervised research and reviewed data. LR and KaK wrote the paper with contributions from all authors.

\section{ACKNOWLEDGMENTS}

The research was funded by the European Union through the European Regional Development Fund (Project No. 20122015.3.2.0701.12-0049 and 2014-2020.4.01.15-0012), by Estonian Research Council grant IUT 2-2, and by personal research grant PUT1367, PUT177, PUT1465 and PUT1669. We thank Prof. Annamari Ranki for critical reading of the manuscript and valuable comments.

\section{SUPPLEMENTARY MATERIAL}

The Supplementary Material for this article can be found online at: https://www.frontiersin.org/articles/10.3389/fimmu. 2018.02707/full\#supplementary-material

7. Singh A, Gotherwal V, Junni P, Vijayan V, Tiwari M, Ganju P, et al. Mapping architectural and transcriptional alterations in non-lesional and lesional epidermis in vitiligo. Sci Rep. (2017) 7:9860. doi: 10.1038/s41598-017-10253-w

8. Moretti S, Fabbri P, Baroni G, Berti S, Bani D, Berti E, et al. Keratinocyte dysfunction in vitiligo epidermis: cytokine microenvironment and correlation to keratinocyte apoptosis. Histol Histopathol. (2009) 24:84957. doi: 10.14670/HH-24.849

9. Richmond JM, Bangari DS, Essien KI, Currimbhoy SD, Groom JR, Pandya AG, et al. Keratinocyte-derived chemokines orchestrate T-cell positioning in the epidermis during vitiligo and may serve as biomarkers of disease. J Invest Dermatol. (2017) 137:350-58. doi: 10.1016/j.jid.2016.09.016

10. Sandoval-Cruz M, Garcia-Carrasco M, Sanchez-Porras R, Mendoza-Pinto C, Jimenez-Hernandez M, Munguia-Realpozo P, et al. Immunopathogenesis of vitiligo. Autoimmun Rev. (2011) 10:762-65. doi: 10.1016/j.autrev.2011.0 2.004

11. LePoole IC, van den Wijngaard R, Westerhof W, Das PK. Presence of T cells and macrophages in inflammatory vitiligo skin parallels melanocyte disappearance. Am J Pathol. (1996) 148:1219-28.

12. Boniface K, Jacquemin C, Darrigade AS, Dessarthe B, Martins C, Boukhedouni $\mathrm{N}$, et al. Vitiligo skin Is imprinted with resident memory 
CD8 T cells expressing CXCR3. J Invest Dermatol. (2018) 138:355-64. doi: 10.1016/j.jid.2017.08.038

13. van den Boorn JG, Konijnenberg D, Dellemijn TA, van der Veen JP, Bos JD, Melief CJ, et al. Autoimmune destruction of skin melanocytes by perilesional T cells from vitiligo patients. J Invest Dermatol. (2009) 129:222032. doi: $10.1038 /$ jid.2009.32

14. Cheuk S, Schlums H, Serezal IG, Martini E, Chiang SC, Marquardt $\mathrm{M}$, et al. CD49a expression defines tissue-resident CD8(+) $\mathrm{T}$ cells poised for cytotoxic function in human skin. Immunity (2017) 46:287300.doi: 10.1016/j.immuni.2017.01.009

15. Richmond JM, Strassner JP, Zapata L, Garg M, Riding RL, Refat MA, et al. Antibody blockade of IL-15 signaling has the potential to durably reverse vitiligo. Sci Transl Med. (2018) 10:eaam7710. doi: $10.1126 /$ scitranslmed.aam 7710

16. Bertolotti A, Boniface K, Vergier B, Mossalayi D, Taieb A, Ezzedine $\mathrm{K}$, et al. and J. Type I interferon signature in the initiation of the immune response in vitiligo. Pigment Cell Melanoma Res. (2014) 27:398-407. doi: $10.1111 /$ pcmr.12219

17. Reemann P, Reimann E, Ilmjarv S, Porosaar O, Silm H, Jaks V, et al. Melanocytes in the skin-comparative whole transcriptome analysis of main skin cell types. PLoS ONE (2014) 9:e115717. doi: 10.1371/journal.pone.0115717

18. Geissmann F, Prost C, Monnet JP, Dy M, Brousse N, Hermine O. Transforming growth factor beta 1 in the presence of granulocyte/macrophage colony-stimulating factor and interleukin 4 , induces differentiation of human peripheral blood monocytes into dendritic Langerhans cells. J Exp Med. (1998) 187:961-6. doi: 10.1084/jem.187.6.961

19. Schindelin J, Arganda-Carreras I, Frise E, Kaynig V, Longair M, Pietzsch T, et al. Fiji: an open-source platform for biological-image analysis. Nat Methods (2012) 9:676-82. doi: 10.1038/nmeth.2019

20. Moretti S, Spallanzani A, Amato L, Hautmann G, Gallerani I, Fabiani $M$, et al. New insights into the pathogenesis of vitiligo: imbalance of epidermal cytokines at sites of lesions. Pigment Cell Res. (2002) 15:87-92. doi: $10.1034 / j .1600-0749.2002 .10049 . x$

21. Grimes PE, Morris R, Avaniss-Aghajani E, Soriano T, Meraz M, Metzger, A. Topical tacrolimus therapy for vitiligo: therapeutic responses and skin messenger RNA expression of proinflammatory cytokines. J Am Acad Dermatol. (2004) 51:52-61. doi: 10.1016/j.jaad.2003.12.031

22. Reimann E, Kingo K, Karelson M, Reemann P, Loite U, Sulakatko $\mathrm{H}$, et al. The mRNA expression profile of cytokines connected to the regulation of melanocyte functioning in vitiligo skin biopsy samples and peripheral blood mononuclear cells. Hum Immunol. (2012) 73:393-8. doi: 10.1016/j.humimm.2012.01.011

23. Regazzetti C, Joly F, Marty C, Rivier M, Mehul B, Reiniche P, et al. Transcriptional analysis of vitiligo skin reveals the alteration of WNT pathway: a promising target for repigmenting vitiligo patients. I Invest Dermatol. (2015) 135:3105-14. doi: 10.1038/jid.2015.335

24. Rashighi M, Agarwal P, Richmond JM, Harris TH, Dresser K, Su MW, et al. CXCL10 is critical for the progression and maintenance of depigmentation in a mouse model of vitiligo. Sci Transl Med. (2014) 6:223ra23. doi: 10.1126/scitranslmed.3007811

25. Rätsep R, Kingo K, Karelson M, Reimann E, Raud K, Silm H, et al. Gene expression study of IL10 family genes in vitiligo skin biopsies, peripheral blood mononuclear cells and sera. Br J Dermatol. (2008) 159:1275-81. doi: 10.1111/j.1365-2133.2008.08785.x

26. Hamilton JA, Cook AD, Tak PP. Anti-colony-stimulating factor therapies for inflammatory and autoimmune diseases. Nat Rev Drug Discov. (2017) 16:53-70. doi: 10.1038/nrd.2016.231

27. Martinet V, Tonon S, Torres D, Azouz A, Nguyen M, Kohler A, et al. Type I interferons regulate eomesodermin expression and the development of unconventional memory CD8(+) T cells. Nat Commun. (2015) 6:7089. doi: $10.1038 /$ ncomms 8089

28. Barbarin A, Cayssials E, Jacomet F, Nunez NG, Basbous S, Lefevre L, et al. Phenotype of NK-like CD8(+) T cells with innate features in humans and their relevance in cancer diseases. Front Immunol. (2017) 8:316. doi: 10.3389/fimmu.2017.00316

29. Ghadially H, Brown L, Lloyd C, Lewis L, Lewis A, Dillon J, et al. MHC class I chain-related protein A and B (MICA and MICB) are predominantly expressed intracellularly in tumour and normal tissue. Br J Cancer (2017) 116:1208-17. doi: 10.1038/bjc.2017.79

30. Tembhre MK, Parihar AS, Sharma VK, Sharma A, Chattopadhyay P, Gupta $\mathrm{S}$. Alteration in regulatory $\mathrm{T}$ cells and programmed cell death 1-expressing regulatory $\mathrm{T}$ cells in active generalized vitiligo and their clinical correlation. Br J Dermatol. (2015) 172:940-50. doi: 10.1111/bjd.13511

31. Ho HA, Kapadia R, Al-Tahan S, Ahmad S, Ganesan AK. WIPI1 coordinates melanogenic gene transcription and melanosome formation via TORC1 inhibition. J Biol Chem. (2011) 286:12509-32. doi: 10.1074/jbc.M110.2 00543

32. Akinduro O, Sully K, Patel A, Robinson DJ, Chikh A, McPhail G, et al. Constitutive autophagy and nucleophagy during epidermal differentiation. $J$ Invest Dermatol. (2016) 136:1460-70. doi: 10.1016/j.jid.2016.03.016

33. Strassner JP, Rashighi M, Refat MA, Richmond JM, Harris JE. Suction blistering the lesional skin of vitiligo patients reveals useful biomarkers of disease activity. J Am Acad Dermatol. (2017) 76:847-55. doi: 10.1016/j.jaad.2016.12.021

34. Wang $\mathrm{XX}$, Wang QQ, Wu JQ, Jiang $\mathrm{M}$, Chen $\mathrm{L}$, Zhang $\mathrm{CF}$, et al. Increased expression of CXCR3 and its ligands in patients with vitiligo and CXCL10 as a potential clinical marker for vitiligo. Br J Dermatol. (2016) 174:1318-26. doi: $10.1111 /$ bjd. 14416

35. Moll HP, Maier T, Zommer A, Lavoie T, Brostjan C. The differential activity of interferon-alpha subtypes is consistent among distinct target genes and cell types. Cytokine (2011) 53:52-9. doi: 10.1016/j.cyto.2010.09.006

36. Jacquemin C, Rambert J, Guillet S, Thiolat D, Boukhedouni N, Doutre MS, et al. Heat shock protein 70 potentiates interferon alpha production by plasmacytoid dendritic cells: relevance for cutaneous lupus and vitiligo pathogenesis. Br J Dermatol. (2017) 177:1367-75. doi: 10.1111/bjd.15550

37. Arya V, Bansal M, Girard L, Arya S, Valluri A. Vitiligo at injection site of PEG-IFN-alpha $2 \mathrm{a}$ in two patients with chronic hepatitis C: case report and literature review. Case Rep Dermatol. (2010) 2:156-64. doi: $10.1159 / 000320207$

38. Crampton SP, Deane JA, Feigenbaum L, Bolland S. Ifih1 gene dose effect reveals MDA5-mediated chronic type I IFN gene signature, viral resistance, and accelerated autoimmunity. J Immunol. (2012) 188:1451-9. doi: 10.4049/jimmunol.1102705

39. Hartlova A, Erttmann SF, Raffi FAM, Schmalz AM, Resch U, Anugula S, et al. DNA damage primes the type I interferon system via the cytosolic DNA sensor STING to promote anti-microbial innate immunity. Immunity (2015) 42:332-43. doi: 10.1016/j.immuni.2015.01.012

40. Jian Z, Li K, Song P, Zhu GN, Zhu LF, Cu TT, et al. Impaired activation of the Nrf2-ARE signaling pathway undermines $\mathrm{H} 2 \mathrm{O} 2$-induced oxidative stress response: a possible mechanism for melanocyte degeneration in vitiligo. $J$ Invest Dermatol. (2014) 134:2221-30. doi: 10.1038/jid.2014.152

41. Shafi S, Vantourout P, Wallace G, Antoun A, Vaughan R, Stanford M, et al. and A. An NKG2D-mediated human lymphoid stress surveillance response with high interindividual variation. Science Translat Med. (2011) 3:113. doi: 10.1126/scitranslmed.3002922

42. Strid J, Sobolev O, Zafirova B, Polic B, Hayday A. The intraepithelial T cell response to NKG2D-ligands links lymphoid stress surveillance to atopy. Science (2011) 334:1293-7. doi: 10.1126/science.1211250

43. Kovacs D, Bastonini E, Ottaviani M, Cota C, Migliano E, Dell'Anna ML, et al. Vitiligo skin: exploring the dermal compartment. J Invest Dermatol. (2018) 138:394-404. doi: 10.1016/j.jid.2017.06.033

44. Yu R, Broady R, Huang YS, Wang Y, Yu J, Gao M, et al. Transcriptome analysis reveals markers of aberrantly activated innate immunity in vitiligo lesional and non-lesional skin. PLoS ONE (2012) 7:e51040. doi: 10.1371/journal.pone.0051040

45. Mohan GC, Silverberg JI. Association of vitiligo and alopecia areata with atopic dermatitis a systematic review and meta-analysis. JAMA Dermatol. (2015) 151:522-8.doi: 10.1001/jamadermatol.2014.3324

46. Harning R, Cui J, Bystryn JC Relation between the incidence and level of pigment cell antibodies and disease-activity in vitiligo. J Invest Dermatol. (1991) 97:1078-80. doi: 10.1111/1523-1747.ep12492607

47. Kemp EH, Gavalas NG, Gawkrodger DJ, Weetman AP. Autoantibody responses to melanocytes in the depigmenting skin disease vitiligo. Autoimmun Rev. (2007) 6:138-42. doi: 10.1016/j.autrev.2006.0 9.010 
48. Yu T, Zuber J, Li JC. Targeting autophagy in skin diseases. J Mol Med. (2015) 93:31-8. doi: 10.1007/s00109-014-1225-3

49. Zhou XJ, Zhang H. Autophagy in immunity Implications in etiology of autoimmune/autoinflammatory diseases. Autophagy (2012) 8:1286-99. doi: 10.4161 /auto. 21212

50. Jeong TJ, Shin MK, Uhm YK, Kim HJ, Chung JH, Lee MH. Association of UVRAG polymorphisms with susceptibility to non-segmental vitiligo in a Korean sample. Exp Dermatol. (2010) 19:E323-5. doi: 10.1111/j.1600-0625.2009.01039.x

51. Wang P, Li Y, Nie HQ, Zhang XY, Shao QY, Hou XL, et al. The changes of gene expression profiling between segmental vitiligo, generalized vitiligo and healthy individual. J Dermatol Sci. (2016) 84:40-9. doi: 10.1016/j.jdermsci.2016. 07.006

52. Zhang CF, Gruber F, Ni CY, Mildner M, Koenig U, Karner S, et al. Suppression of autophagy dysregulates the antioxidant response and causes premature senescence of melanocytes. J Invest Dermatol. (2015) 135:1348-57. doi: 10.1038/jid.2014.439

53. Xie H, Zhou FB, Liu L, Zhu GN, Li Q, Li C, et al. Vitiligo: How do oxidative stress-induced autoantigens trigger autoimmunity? J Dermatol Sci. (2016) 81:3-9. doi: 10.1016/j.jdermsci.2015.09.003
54. Yun WJ, Kim EY, Park JE, Jo SY, Bang SH, Chang EJ, et al. Microtubuleassociated protein light chain 3 is involved in melanogenesis via regulation of MITF expression in melanocytes. Sci Rep. (2016) 6:19914. doi: $10.1038 /$ srep 19914

55. Tobin DJ, Swanson NN, Pittelkow MR, Peters EM, Schallreuter KU. Melanocytes are not absent in lesional skin of long duration vitiligo. J Pathol (2000) 191:407-16. doi: 10.1002/1096-9896(2000)9999:9999<:: AID-PATH659>3.0.CO;2-D

Conflict of Interest Statement: The authors declare that the research was conducted in the absence of any commercial or financial relationships that could be construed as a potential conflict of interest.

Copyright (c) 2018 Raam, Kaleviste, Šunina, Vaher, Saare, Prans, Pihlap, Abram, Karelson, Peterson, Rebane, Kisand and Kingo. This is an open-access article distributed under the terms of the Creative Commons Attribution License (CC BY). The use, distribution or reproduction in other forums is permitted, provided the original author(s) and the copyright owner(s) are credited and that the original publication in this journal is cited, in accordance with accepted academic practice. No use, distribution or reproduction is permitted which does not comply with these terms. 